\title{
Antitumor activity of neurokinin-1 receptor antagonists in MG-63 human osteosarcoma xenografts
}

\author{
MIGUEL MUÑOZ ${ }^{1}$, MICHAEL BERGER ${ }^{2,3}$, MARISA ROSSO $^{1}$, ANA GONZALEZ-ORTEGA ${ }^{1}$, \\ ANDRÉS CARRANZA ${ }^{4}$ and RAFAEL COVENAS ${ }^{5}$ \\ ${ }^{1}$ Virgen del Rocío University Hospital, Research Laboratory on Neuropeptides; ${ }^{2}$ Division of Pediatric Infectious Disease \\ and Immunopathology, Virgen del Rocío Children's Hospital, Institute of Biomedicine, Seville, Spain; \\ ${ }^{3}$ Department of Pediatric Surgery, Research Laboratories, Dr. von Hauner Children's Hospital, \\ Ludwig-Maximilians-University, Munich, Germany; ${ }^{4}$ Deparment of Pathology, \\ Virgen del Rocío University Hospital, Seville; ${ }^{5}$ Laboratory of Neuroanatomy of the Peptidergic Systems, \\ Institute of Neurosciences of Castilla y León, Salamanca, Spain
}

Received August 8, 2013; Accepted October 1, 2013

DOI: $10.3892 /$ ijo.2013.2164

\begin{abstract}
Osteosarcoma is a highly malignant bone tumor in children and adolescents. Aprepitant is a selective high-affinity antagonist of the human neurokinin-1 (NK-1) receptor (NK1R) with robust antitumor activity. No data exist on the presence of NK1R in osteosarcoma and whether this tumor responds to NK1R antagonists. Here, we analyzed the expression of NK1R in the human osteosarcoma cell line MG-63 with western blot analysis and PCR and found significant expression both at the protein and mRNA levels. We further studied the growth inhibitory capacity of aprepitant and other NK1R antagonists on MG-63 in vitro using an MTS cytotoxicity assay and DAPI staining. All antagonists induced tumor growth inhibition and apoptosis. Synergism was observed for the combination of L-733,060 with common cytostatic drugs in MG-63, but not in non-malignant HEK 293 cells. Pretreatment of HEK293 with L-733,060 prior to exposure to cytostatic drugs partially protected HEK293 cells from inhibition by these drugs. Furthermore, nanomolar concentrations of substance P (SP), the natural ligand of the NK1R, increased the growth rate of MG-63 cells and micromolar concentrations of aprepitant inhibited SP-induced growth in a dose-dependent manner. In vivo, a xenograft for MG-63 was created in nude mice and treated with peritumoral s.c. injections of fosaprepitant, which resulted in a significant reduction of tumor volume. Collectively, we demonstrated for the first time that the NK1R
\end{abstract}

Correspondence to: Dr Miguel Muñoz, Hospital Infantil Virgen del Rocío, Laboratorio de Neuropéptidos, Av. Manuel Siurot s/n, Seville 41013, Spain

E-mail:mmunoz@cica.es

Key words: neurokinin-1 receptor, aprepitant, substance P, antitumor target, osteosarcoma, tumor cell lines, xenograft, L-733,060, L-732,138, apoptosis, fosaprepitant is expressed in human osteosarcoma cell line MG-63 and that this receptor can be targeted with NK1R antagonists both in vitro as well as in vivo.

\section{Introduction}

Osteosarcoma is a high-grade bone neoplasm that originates from mesenchymal tissue. It is the third most common malignancy in children and adolescents, the most frequent highly malignant bone-tumor and reaches a peak manifestation during the second and third decade of life. Although survival rates increased up to $70 \%$ in patients with localized disease, non-responsiveness to chemotherapy is repeatedly encountered and paired with poor outcome (1). Currently, the treatment of osteosarcoma involves surgery with pre- and postoperative chemotherapy including multiple chemotherapeutic agents. If metastases are present, results are poor with long-term survival of less than $20 \%$ (2).

The neurokinin-1 (NK-1) receptor (NK1R) has recently been discovered to play an integral role in the maintenance of a favorable tumor microenvironment $(3,4)$. The NK1R is a tachykinin receptor; its natural ligand is substance P (SP). Tachykinins belong to the family of neuropeptides that are responsible for a myriad of physiological responses. They bind to specific seven transmembrane, $G$ protein-coupled receptors (GPCRs) on target cells. Three mammalian tachykinin receptor subtypes have been characterized, TACR1 (NK1R), TACR2 and TACR3, which show preferential but not absolute selectivity for SP, neurokinin A (NK-A) and neurokinin $\mathrm{B}$ (NK-B), respectively $(5,6)$. Tachykinin receptors are expressed by many different cell types and respond to tachykinins in a cell type-specific manner. These receptors are linked to a variety of physiological and biological processes such as regulation of neurotransmission, pain, inflammation, cell growth and differentiation as well as oncogenesis, among others $(3,4)$.

SP and the subsequent activation of NK1R (SP/NK-receptor complex) strongly influence the tumor microenvironment $(3,4)$. 
Activation of NK1R by SP leads to phosphoinositide hydrolysis, calcium mobilization and mitogen-activated protein kinase (MAPK) activation (7-10). Moreover, the activation of the NK1R was found to be involved in a myriad of processes related to oncogenesis such as mitogenesis, angiogenesis, cell migration and metastasis. In this sense, it has been demonstrated that SP acts through the NK1R as a mitogen in several human cancer cell lines, including astrocytoma, melanoma, neuroblastoma, glioma, retinoblastoma as well as pancreatic, larynx, colon and gastric carcinoma, leukemia and breast cancer (11-18). In these cell lines, the pharmaceutical blockage of this receptor was found to robustly inhibit tumor growth, making this receptor an attractive target for future anticancer strategies (4).

Aprepitant is a selective high-affinity antagonist of the human NK-1 receptor. Currently, this drug is used in clinical practice as an antiemetic (19-22). Additionally, in an experimental setting, it was found to have great potential as an antitumor agent for a broad spectrum of cancers (20).

Other NK1R antagonist, such as L-733,060 (a piperidine derivative) and L-732,138 show affinity for the human NK1R in vitro (14). Both induce antitumor activity in human cancer cell lines $(4,12-15,23,24)$.

It has been demonstrated in in vitro studies that coadministration of L-733,060 with vinblastine or microtubuledestabilizing agents resulted in synergistic inhibition of cancer cell growth in cells that express NK1R. Interestingly, this effect was not observed in non-cancerous cells $(3,25)$.

Moreover, measuring the growth inhibition of MDA-MB-231 tumor cell xenografts the antitumor activity of NK1R and NK-2 receptor (NK2R) antagonists has been demonstrated in nude mice $(26,27)$.

However, nothing is known regarding the expression of the NK1R in osteosarcomas and whether this receptor can serve as a target for future anticancer strategies against this tumor, either alone or together with cytostatic drugs. Likewise, no data exist regarding a potential therapeutic effect of NK1R antagonists against osteosarcoma in vivo. Therefore, in this study we investigated the role of the NK1R as well as its therapeutic effect in a human osteosarcoma cell line (MG-63) in vitro and in a mouse xenograft in vivo.

\section{Materials and methods}

Cell culture. MG-63 osteosarcoma and other cell lines (HEK293, GAMG, MRC-5) were purchased from European Collection of Cell Cultures (ECACC). We used standard DMEM medium with $10 \%$ FCS, $1 \%$ glutamine and $1 \%$ pentamycin/streptomycin (medium and all supplements from Life Technologies) for all cell lines except HEK293. HEK293 cell line was cultured in DMEM containing additional glutamine. All cell lines were cultivated in either 25 or $75 \mathrm{~cm}^{2}$ culture flasks and incubated at $37^{\circ} \mathrm{C}$ in a humidified atmosphere of $95 \%$ air $/ 5 \% \mathrm{CO}_{2}$ according to the manufacturer's instruction and exclusively grew in adherent monolayers.

Primary human fibroblasts were isolated from skin biopsies from health adult donors. Informed consent was obtained prior to all biopsies and reviewed by the ethics committee of our hospital. Cells were cultured in a similar way as described for cell lines and grew in adherent monolayers exclusively. Medium was DMEM containing $10 \%$ FCS, $1 \%$ glutamine and
$1 \%$ pentamycin/streptomycin (medium and all supplements from Life Technologies). Experiments were carried out prior to 10 passages in all cases.

Drug treatments. The NK1R antagonist aprepitant [MW 534.43 (5-[[(2R, 3S)-2-[(1R)-1-[3,5-bis(trifluoromethyl)phenyl] ethoxy]-3-(4-fluorophenyl)-4-morpholinyl]methyl]-1,2-dihydro3H-1,2,4-triazol-3-one)) was obtained from Merck Research Laboratories (Madrid, Spain), and was dissolved in distilled water containing $0.1 \%$ acetonitrile before sample treatment. In order to determine the $\mathrm{IC}_{50}$, different concentrations $(5$ to $80 \mu \mathrm{M})$ of aprepitant were evaluated. Fosaprepitant dimeglumin is a lyophilized prodrug of aprepitant and is chemically described as 1-Deoxy-1(methylamino)-D-glucitol[3-[[(2R,3S)-2-[(1R)-1[3,5bis(trifluoromethyl)phenyl]ethoxy]-3-(4-fluorophenyl) 4-morpholinyl]methyl]-2,5-dihydro-5-oxo-1H-1,2,4-triazol-1yl]phosphonate (2:1) salt. It was purchased from Merck Sharp $\&$ Dohme. Fosaprepitant was dissolved and diluted according to the manufacturer's instruction.

The NK1R antagonist N-acetyl-L-tryptophan 3, 5-bis (trifluoromethyl) benzyl ester, molecular weight 472.39 (L-732,138; Sigma-Aldrich, Madrid, Spain) and (2S,3S)-3-\{[3,5-bis(trifluoromethyl)benzyl]oxy\}-2-phenylpiperidine, molecular weight 403.36 (L-733,060: Sigma-Aldrich), was dissolved in distilled water containing $2.5 \%$ dimethyl sulfoxide (DMSO) before sample treatment.

SP and acetate salt (Sigma-Aldrich) were dissolved in distilled water and different concentrations (5, 10, 50, 100 and $500 \mathrm{nM}$ ) were used. In experiments investigating synergistic or competing effects, the MG-63 cell line was incubated with SP for $1 \mathrm{~h}$ before the addition of other stimuli.

The common cytostatics adriamycin, mitomycin, ifosfamide and cisplatin were supplied by the general farmacy of the Virgen del Rocío Children's Hospital and were used at the doses indicated in the text and figure legends.

Animals. Female athymic nu/nu nude mice, 5-7-week-old were purchased from Harlan Iberia (Barcelona, Spain), maintained in mircoisolator cages and supplied with sterile materials and food. Permission for animal experiments in the described setting was obtained from the ethics committee of Virgen del Rocío University Hospital (Seville, Spain).

Proliferation assays. Cell proliferation was evaluated using the tetrazolium compound 3-(4,5-dimethylthiazol-2-yl)5-(3-carboxymethoxyphenyl)2-(4-sulfophenyl)-2H-tetrazolium, inner salt (MTS), according to the manufacturer's instructions (CellTiter 96 Aqueous One-Solution Cell Proliferation Assay, Promega Corp., Madison, WI, USA). Cell numbers were quantified using a Coulter counter. The plate included blank wells $(0$ cells $/ 0.1 \mathrm{ml})$, control wells $\left(10^{4}\right.$ cells $\left./ 0.1 \mathrm{ml}\right)$, control wells with acetonitrile, control wells treated with aprepitant and control wells treated with the most effective SP concentration and aprepitant. The plates were inoculated with aprepitant (5-80 $\mu \mathrm{M}$ for tumor cell lines) and were incubated for the first doubling time specific for each tumor cell line. Plates were also inoculated with the most mitogenic exogenous SP nM concentration with or without the $50 \% \mu \mathrm{M}$ inhibition concentration $\left(\mathrm{IC}_{50}\right)$ of aprepitant for their first doubling times. For the proliferation assay, $20 \mu \mathrm{l}$ of the MTS reagent was added to 
each well 90 min before reading the samples on a multiscanner microplate reader (Tecan Spectra Classic, Barcelona, Spain) at $492 \mathrm{~nm}$. Each experimental condition was assayed in duplicate and all experiments were performed at least three times.

For experiments analyzing synergistic effects in MG-63 and HEK293 cell lines, cytostatics and NK1R antagonists were co-cultured at the doses described in the text. For pretreatment experiments of HEK293, cells were pre-incubated for $24 \mathrm{~h}$ with NK1R antagonist L-733,060. After this, cisplatin and ifosfamide were added for an additional $24 \mathrm{~h}$ and cytotoxicity assay was conducted as described.

DAPI staining. In order to determine whether apoptosis was induced, DAPI staining was performed. Briefly, after treatment with aprepitant for their first doubling times, the cells were fixed in $4 \%$ paraformaldehyde. Following a second wash in PBS, cells were incubated in DAPI solution (Sigma-Aldrich) at a dilution of 1/1,000 $(1 \mu \mathrm{g} / \mathrm{ml})$ for $30 \mathrm{~min}$ in the dark. Cells were then analyzed through a fluorescence microscope (Zeiss, Oberkochen, Germany). Apoptotic cells were defined by chromatin condensation and nuclear fragmentation. We counted the number of apoptotic cells, repeating the counts on three different slides. Finally, on each slide we counted the number of apoptotic cells located in five different sequential fields.

Western blot analyses. Total protein was prepared from cell cultures from MG-63, GAMG, MRC-5 and HEK293 cell lines as previously reported (13). Protein concentrations were determined using the protein assay kit from Bio-Rad according to the manufacturer's instructions. From each sample, $40 \mu \mathrm{g}$ of protein was separated by electrophoresis on $12 \%$ SDS-polyacrylamide gels and electroblotted onto PVDF membranes. Blots were incubated in blocking solution [5\% non-fat milk in PBS, 0.1\% Tween-20 (PBS-T)], followed by overnight incubation with an antibody against the KTMTESSSFYSNMLA conserved domain, corresponding to the C-terminus of the NK1R (Sigma-Aldrich) and diluted 1:4,000. Membranes were then washed with PBS-T and incubated with a horseradish peroxidase-conjugated goat anti-rabbit IgG antibody for $2 \mathrm{~h}$ at room temperature $(1: 10,000$ dilution). Antibody detection was performed with an enhanced chemiluminescence reaction (ECL Western blotting detection; Amersham Life Science, Amersham, UK). Pan-cadherin and the secondary antibody alone served as a control.

Polymerase chain reaction (PCR). Real-time PCR was carried out for the cell lines MG-63, GAMG and HEK293 as well as primary human fibroblasts.

RNA extraction and reverse transcription. From cultured cells, total RNA isolation was obtained after using the NucleoSpin RNA II Kit (Macherey-Nagel), allowing approximately the purification of $5 \times 10^{6}$ cultured cells. Final RNA was dissolved in RNase-free water. The purity and quality of the RNA were determined with Nanodrop ${ }^{\circledR}$. Reverse transcription with elimination of genomic DNA was performed according to the manufacturer's instructions (QuantiTect Reverse transcription Handbook, Qiagen). We carried out all the reactions on ice in order to minimize the risk of RNA degradation. The cDNA obtained was kept at $-80^{\circ} \mathrm{C}$.
Amplification. From the cDNA preparation, $2 \mu \mathrm{l}$ was used in PCR with specifics primers (according to the modified method of Bigioni et al (27) based on the common sequence of the TAC1 human isoforms (NM 001058, NM 015727) TAC1-F (CTG CTG GTG ATT GGC TAT GC) and TAC1-R (AGG AGG AAG AAG ATG TGG AAG G) which yield a 186 bp fragment (28). The amplification of the specimen was performed in a final reaction volume of $20 \mu \mathrm{l}$, and was incubated at $95^{\circ} \mathrm{C}$ for $7 \mathrm{~min}$, subjected to 40 cycles of $95^{\circ} \mathrm{C}$ for $30 \mathrm{sec}, 60^{\circ} \mathrm{C}$ for $30 \mathrm{sec}$ and $72^{\circ} \mathrm{C}$ for $30 \mathrm{sec}$, followed by a final extension cycle at $72^{\circ} \mathrm{C}$ for $7 \mathrm{~min}$. The amplification products were visualized by electrophoresis on $2 \%$ agarose gel stained with ethidium bromide.

Real-time quantitative RT-PCR. The PCR was performed with the LightCycler FastStart DNA SYBR-Green I kit (Roche) according to the protocol provided in the parameter-specific kits and as described previously (12). The transcript numbers were normalized according to the expression of $\beta$-actin per microliter of cDNA.

Osteosarcoma xenograft. A human osteosarcoma tumor xenograft was generated from subcutaneous (s.c.) in vivo injection of human MG-63 osteosarcoma cell line $\left(20 \times 10^{6}\right.$ cells/flank $\left./ 0.2 \mathrm{ml}\right)$ in the right flank of adult athymic female nude mice. Five days after tumor cell inoculation MG-63 tumor-bearing mice were randomly divided into treatment and control group. For treatments, all s.c. injections of fosaprepitant were placed at about $5 \mathrm{~mm}$ from the tumor. Fosaprepitant was administered at doses that have been previously shown to produce a complete blockade of tachykinin NK-1-mediated $\mathrm{IC}_{100}$ response maximum inhibition. Tumor growth was followed by caliber measurement of length and width twice weekly. Tumor volume (TV) was calculated by using the formula: volume $\left(\mathrm{mm}^{3}\right)=$ width $^{2} \mathrm{x}$ length $/ 2$. Fosaprepitant was administered s.c. at doses of $80 \mathrm{mg} / \mathrm{kg}$. Treatment intervals were once weekly. At the end of each experiment, tumors were excised and fixed in formalin (37\%) for histological analyses.

Histology. Sections of $3 \mu \mathrm{m}$ of the tumor were stained with hematoxylin and eosin. Then, tumor slides were blinded and interpreted separately by a trained pathologist.

Statistical analyses. Results are expressed as the mean \pm standard error of the mean (SEM). All statistical comparisons were made with a standard t-test and Mann-Whitney U test, with significance at $\mathrm{p}<0.05$ using biostatistics software from GraphPad Prism ${ }^{\circledR}$ (La Jolla, CA, USA). The criterion for significance was ${ }^{*} \mathrm{p}<0.05$ and ${ }^{* *} \mathrm{p}<0.01$ for all comparisons.

\section{Results}

$N K-1$ receptor expression in human $M G-63$ osteosarcoma cell line. In order to identify the expression of NK1R in the human MG-63 osteosarcoma cell line, we performed both RT-PCR and western blot analyses. RT-PCR was carried out as described and expression was detected for mRNA encoding the NK1R. $\beta$-actin functioned as a housekeeping gene. We compared expression levels for human fibroblasts, GAMG cell line, HEK293 and MG-63. Relative gene expression levels for 
A

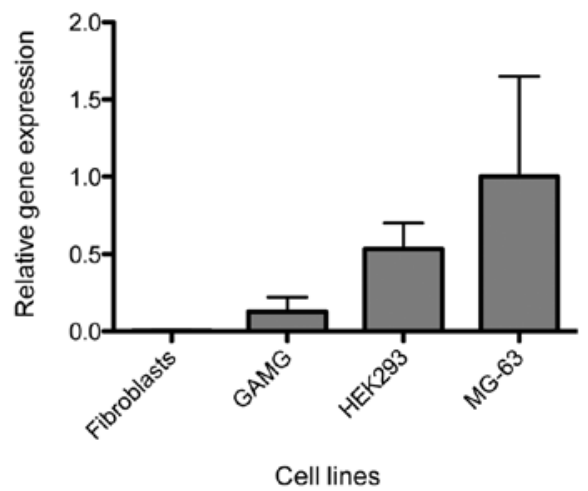

C

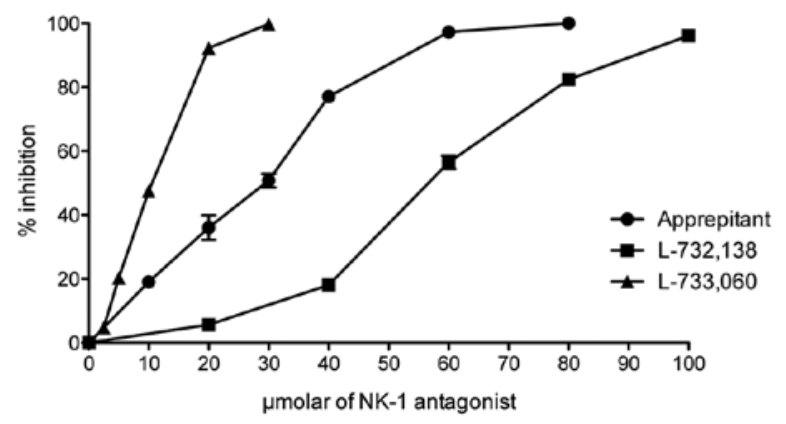

B

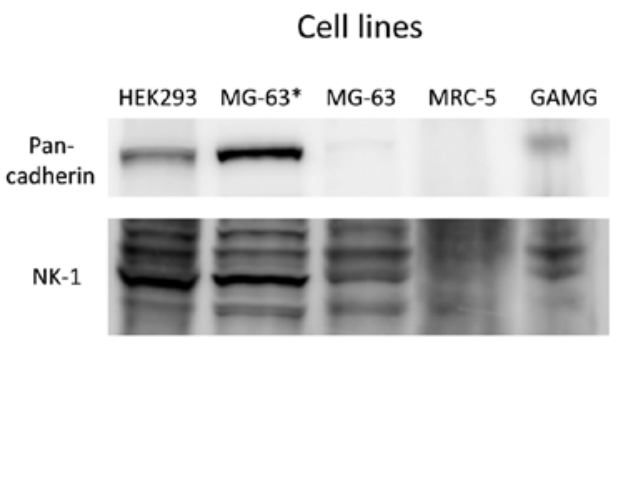

D

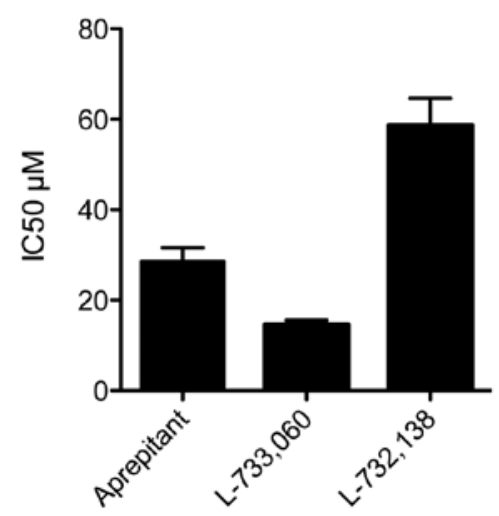

Figure 1. Expression of the NK-1 receptor and growth inhibition in the human osteosarcoma cell line MG-63. (A) mRNA expression for the NK-1 receptor is shown as relative gene expression. RNA was isolated from corresponding cell lines and retro-transcribed to cDNA as described in Materials and methods. Analysis was carried out with RT-PCR, normalization was with the housekeeping gene $\beta$-actin. (B) Protein was extracted and western blot analysis was performed as described. Pan-cadherin served as a control. (C) Cells were cultured in 96-well flat bottom plates and stimulated. Percentage of growth inhibition of the human osteosarcoma cell line MG-63 at $48 \mathrm{~h}$ cells in vitro cultures are shown following the addition of increasing concentrations of L-733,060, L-732,138 and aprepitant. (D) IC ${ }_{50}$ is shown in $\mu \mathrm{M}$ for the three antagonists. Values are means \pm SEM (bars).

NK1R were highest in MG-63, followed by HEK293 with levels of roughly $50 \%$ of what was observed in MG-63. NK1R copies in GAMG were 0.2 compared to MG-63, and human fibroblasts expressed only marginal amounts of NK1R (Fig. 1A).

For western blot analysis, total cell protein extracts were loaded onto polyacrylamide gels, resolved and transferred to membranes as described in Materials and methods. After incubation with the specific antibody, we observed strong expression of the NK1R at $46 \mathrm{kDa}$ in MG-63 and HEK293 cell line (Fig. 1B). Slight expression was found for the GAMG cell line, and essentially no expression could be detected for the fibroblast-like cell line MRC-5. Therefore, the observations made for the expression of mRNA correlated with protein levels analyzed by western blotting. Pan-cadherin antibody served as a control.

Growth inhibition of NK-1 receptor antagonists in the MG-63 cell line. Growth inhibition of the MG-63 cell line by aprepitant, L-733,060 and L-732,138 was analyzed with MTS proliferation assay. MG-63 was incubated with increasing concentrations of all three NK1R antagonists for $48 \mathrm{~h}$. We observed a concentrationdependent growth inhibition for all three antagonists (Fig. 1C). L-733,060 showed the strongest effect and reached $47.45 \%$ growth inhibition at $10 \mu \mathrm{M}$ and $99.78 \%$ growth inhibition at $30 \mu \mathrm{M}$. L-732,138 showed the weakest antitumor effect, resulting in $56.43 \%$ growth inhibition at $60 \mu \mathrm{M}$ and $96.17 \%$ at $100 \mu \mathrm{M}$. Aprepitant showed robust growth inhibition, with 50.78\% inhibition at $30 \mu \mathrm{M}$ and $97.25 \%$ at $60 \mu \mathrm{M}$. Thus, the concentrations required for a $50 \%$ reduction in optical density $\left(\mathrm{IC}_{50}\right)$ observed in the controls treated with aprepitant, L-733,060 and L-732,138 were $28.65,14.55$ and $58.61 \mu \mathrm{M}$ for $48 \mathrm{~h}$, respectively (Fig. 1D).

NK-1 receptor antagonists induce apoptosis of $M G-63$ cell line. After having confirmed substantial growth inhibition of MG-63 cell line with NK1R antagonists, the cell line was cultured with NK1R antagonists and stained with DAPI in order to determine the rate of induction of apoptosis. After administration of either NK1R antagonist L-733,060 or L-732,138, a significant number of apoptotic cells was found in the MG-63 cell line (Fig. 2). After treatment with antagonist L-733,060 at $\mathrm{IC}_{50}$ dose, we observed 34.3 \pm 2.5 (SEM) living cells per high power field (PF) compared to $102.25 \pm 15.6$ in the control. Of these, none were apoptotic in the control (0\%). Treatment with L-733,060 lead to $27.7 \pm 1.69 \%$ apoptotic cells (Fig. 2A-C). After administration of L-732,138, we found a total cell number of $20.25 \pm 5.1$ and $126 \pm 13.0$ for the control. Again, no apoptotic cells were found 
A

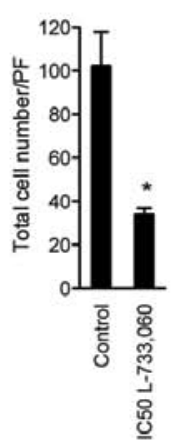

D

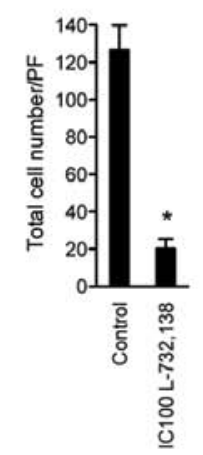

B

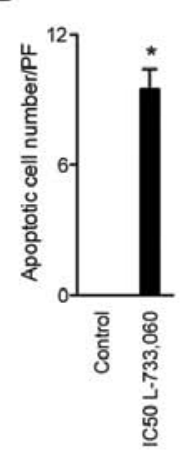

E

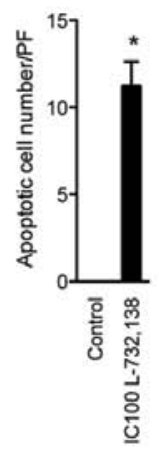

C

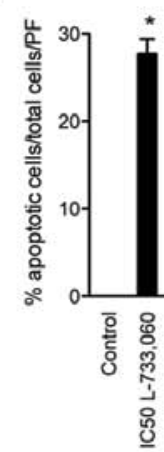

F

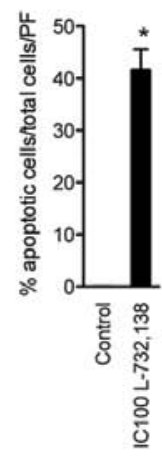

G
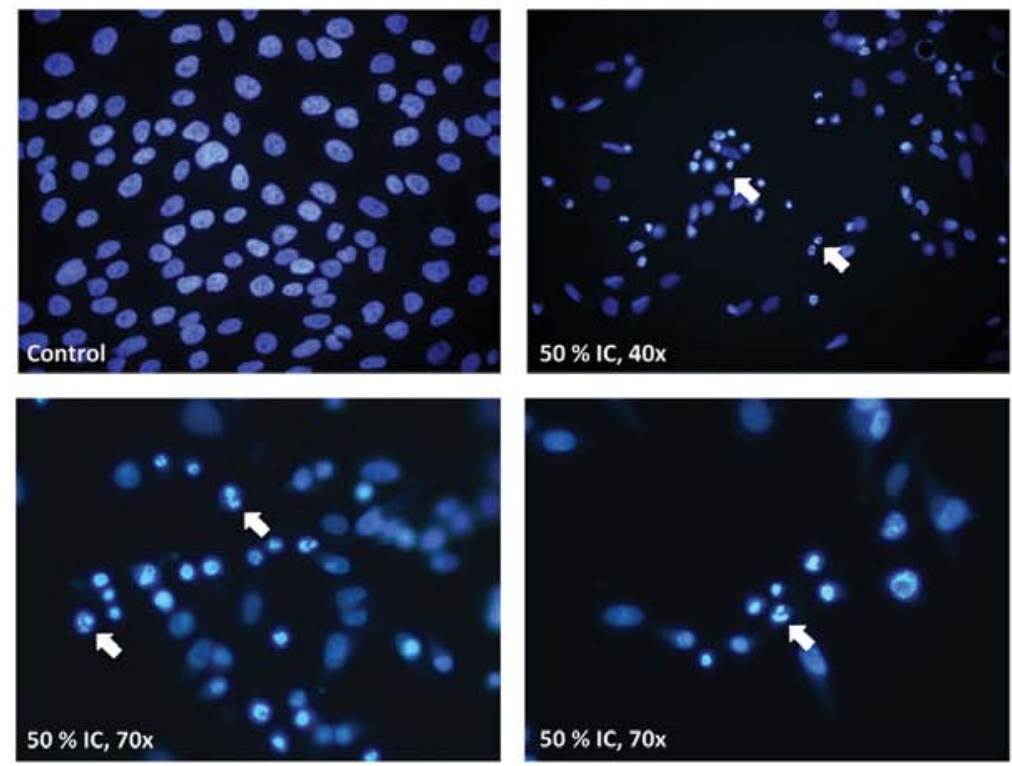

Figure 2. DAPI staining of the human osteosarcoma cell line MG-63. Cells were cultured in 6-well flat bottom plates and stimulated either with (A-C) L-733,060 or (D-F) 732,138. After 48 h, DAPI stain was carried out as described. (A and D) Total cell number is shown per power field (PF). (B and E) The total number of apoptotic cells is shown per PF. (C and F) The percentage of apoptotic cells in relation to all cells per PF is shown. (G) Example slides are shown for the induction of apoptosis with L-733,060. Numerous apoptotic figures of the treated cells can be observed: note the chromatin condensation and the nuclear fragmentation (white arrows, H: x40, right upper panel). Higher-power magnifications are shown in the lower panels (x70). The control is shown at x40 magnification (left upper panel), and cells are mostly intact.

in the control group, but $41.66 \pm 4.0 \%$ apoptotic cells were found after treatment with the antagonist (Fig. 2D-F).

The NK-1 receptor antagonists $L-733,060$ and L-732,138 block substance P-induced mitogenic stimulation. Knowing that SP, the natural ligand for the NK1R, acts as a mitogen in other cell lines, we investigated whether this was also true for the MG-63 cell line. We stimulated cell cultures with increasing concentrations of SP and observed that nanomolar concentrations of SP induced cell proliferation as compared to the untreated controls (Fig. 3A). Even though small, cell proliferation induced by SP stimulation was evident at $5 \mathrm{nM}$ $(6.56 \%)$ and then continuously increased to maximum level of $12.77 \%$ in cellular density at $500 \mathrm{nM}$. Hence, the activation of NK1R with its natural ligand SP leads to proliferation in the human osteosarcoma MG-63 cell line.

We then analyzed whether blockage of NK1R with antagonists could reverse the SP-induced cell proliferation. In these experiments, we stimulated MG-63 cell line with 100 and $500 \mathrm{nM}$ SP and blocked the NK1R with different concentrations of NK1R antagonists L-733,060 and L-732,138. We observed that the mitogenic action of SP can be reversed in the MG-63 cell line with NK1R blockage at $\mathrm{IC}_{50}$ concentrations even at high stimulatory doses of SP [stimulation with $500 \mathrm{nM}$ SP and blockage with $\left.\mathrm{IC}_{50}(60 \mu \mathrm{M}) \mathrm{L}-732,138\right]$ but not if NK1R antagonist dose is negligible (stimulation with
$500 \mathrm{nM}$ SP and blockage with $10 \mu \mathrm{M}$ L-733,060). Therefore, since L-733,060- and L-733,138-induced growth inhibition was partially reversed by the administration of nanomolar doses of exogenous SP, these results indicate that both L-733,060 and L-732,138 block the SP mitogenic stimulation.

Synergism of NK-1 receptor antagonists with common cytostatics. After having observed robust antiproliferative and proapoptotic effects of NK1R antagonists, we studied how this effect compared to common cytostatic drugs (Fig. 3B-E). In an in vitro cell culture experiment, MG-63 cell line was cultured with $\mathrm{L}-733,060$ alone at $\mathrm{IC}_{50}$ or together with $10 \mu \mathrm{M}$ of the cytostatic drugs adriamycin, mitomycin, ifosfamide or cisplatin. In these particular experiments, the mean (with SEM) of four separate experiments was analyzed. Our calculated $\mathrm{IC}_{50}$ dose for L-733,060 induced 45.4 $2.6 \%$ SEM cell inhibition and served as a control for reference throughout this experiment. In the case of adriamycin, we observed $57.4 \pm 2.36 \%$ cell inhibition for the cytostatic drug alone, but $65.0 \pm 1.22 \%$ together with the antagonist at $\mathrm{IC}_{50}$ dose $(\mathrm{p}=0.0292)$. The cell inhibition induced by mitomycin was similar to the cell inhibition observed for the NK1R antagonist alone $(45.4 \pm 2.6 \%$ and $45.1 \pm 0.9 \%$, respectively). Again, a synergistic effect was observed when the cytostatic drug was combined with the NK1R antagonist $(54.2 \pm 3.1, \mathrm{p}=0.0305)$. As observed with adriamycin, cisplatin induced more cell inhibition than the NK1R antagonist alone 

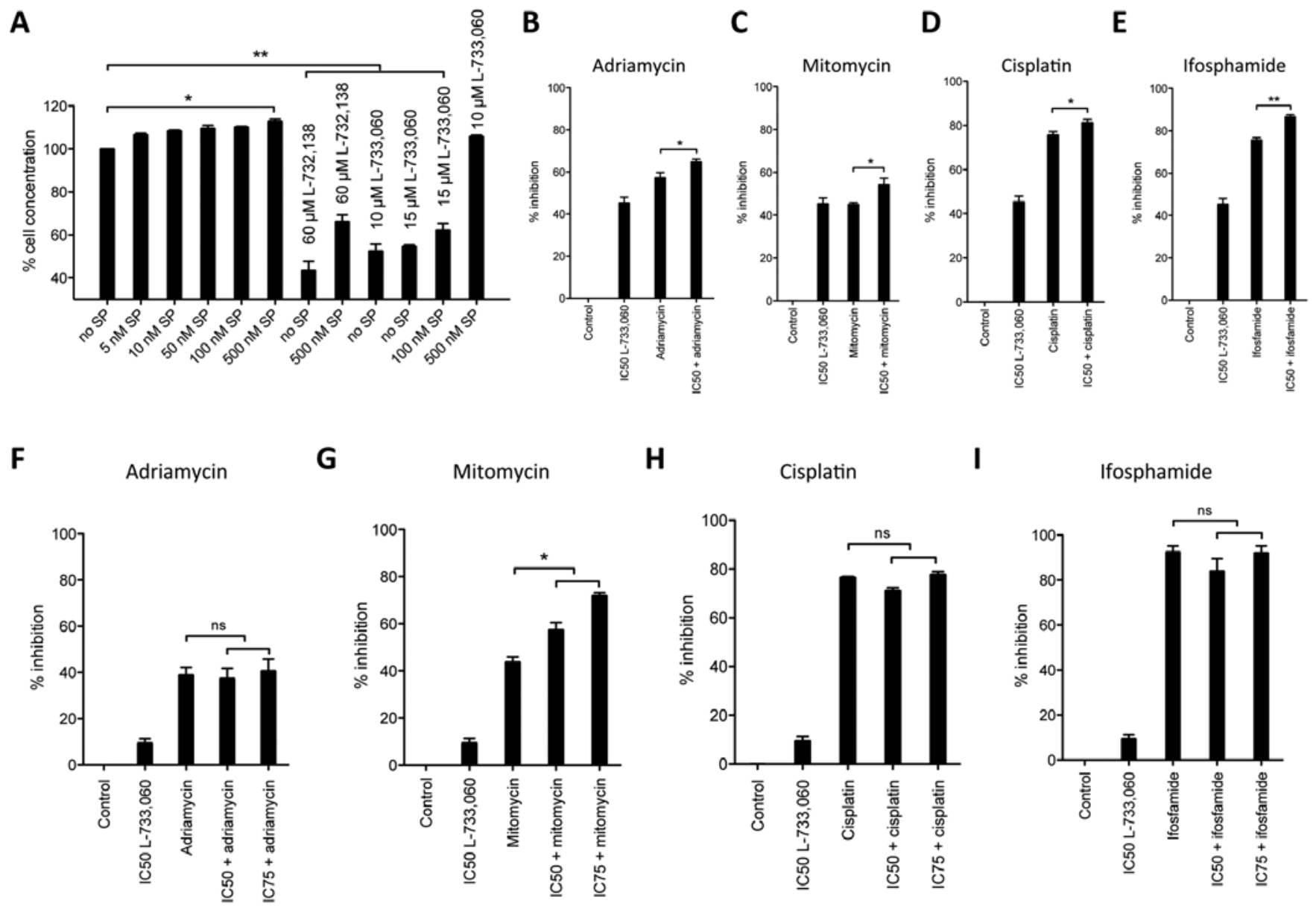

G

H

Cisplatin

I

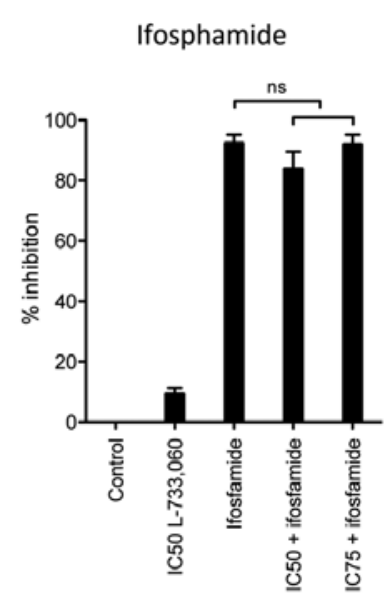

Figure 3. Interaction of MG-63 and HEK293 with SP and cytostatics. (A) Cell growth is shown for the MG-63 cell line after stimulation with different concentrations with SP. Experiments were realized in 96-well flat bottom wells with 10,000 cells per well as described above, and NK-1 antagonists were given together with SP as indicated. (B-E) Cell inhibition is shown for MG-63 cell line for the NK-1 receptor antagonist L-733,060 together with $10 \mu \mathrm{M}$ of (B) adriamycin, (C) mitomycin, (D) cisplatin and (E) ifosfamide. The NK-1 antagonist was given at $\mathrm{IC}_{50}$. (F-I) Cell inhibition is shown for HEK293 cell line for the NK-1 receptor antagonist L-733,060 together with $10 \mu \mathrm{M}$ of $(\mathrm{F})$ adriamycin, $(\mathrm{G})$ mitomycin, $(\mathrm{H})$ cisplatin and (I) ifosfamide. The NK-1 antagonist was given at $\mathrm{IC}_{50}$ and $\mathrm{IC}_{75}$ as indicated in the figure. Error bars represent the SEM. ${ }^{*} \mathrm{p}<0.05 ;{ }^{* *} \mathrm{p}<0.01$ indicate statistical significance.

(75.7 $\pm 2.6 \%$ and $45.4 \pm 2.6 \%$, respectively). Combining the NK1R antagonist and cisplatin induced $81.36 \pm 1.4 \%$ cell inhibition ( $\mathrm{p}=0.0349)$. Ifosfamide induced $75.7 \pm 1.1 \%$ cell inhibition when given alone and $86.66 \pm 1.0 \%$ when given together with the NK1R antagonist, again showing an increased effect $(\mathrm{p}=0.030)$.

Effect of NK-1 receptor antagonists on non-malignant HEK293 cells. Given the robust inhibitory effect observed in MG-63 cell line we assessed whether this inhibition could be unfolded in non-malignant cells as well. We incubated HEK293 cells with NK1R antagonist L-733,060 and common cytostatic drugs and measured cytotoxicity as described (Fig. 3F-I). We intentionally did not recalculate the $\mathrm{IC}_{50}$ and $\mathrm{IC}_{75}$ dosage for HEK293, but used the same dosage we had calculated for MG-63 cells. While observing cell inhibition of only $9.4 \pm 1.8 \%$ for the NK1R antagonist in these cells, we observed robust cell inhibition by the cytostatic drugs in the range of $39-92.1 \%$ (adriamycin $38.9 \pm 3.35 \%$, mitomycin $43.8 \pm 2.17 \%$, cisplatin $76.6 \pm 0.3 \%$ and ifosfamide $92.1 \pm 3.1 \%$, respectively). However, in contrast to what we observed in the malignant MG-63 cell line, when combined with NK1R antagonists, in HEK293 cytostatic drugs induced similar (for adriamycin and mitomycin, Fig. 3F and G) or less (for cisplatin and ifosfamide, Fig. 3H and I) cell inhibition compared to the cytostatic applied alone $\left(\mathrm{IC}_{50} \mathrm{~L}-733,060\right.$ with adriamycin $37.4 \pm 4.41 \%$, mitomycin $57.4 \pm 3.0 \%$, cisplatin $71.2 \pm 1.1 \%$ and ifosfamide $84.0 \pm 5.6 \%$ ). Therefore, importantly, with the exception of mitomycin, no increased effects between the NK1R antagonists used at either $\mathrm{IC}_{50}$ or $\mathrm{IC}_{75}$ and the cytostatic drugs were observed in non-malignant HEK293 cells.

Given the effect observed for L-733,060 in HEK293 cells, in a further experiment, instead of simultaneous incubation with the NK1R antagonist and the respective cytostatic drug, we pre-incubated HEK 293 cells for $24 \mathrm{~h}$ with NK1R antagonist L-733,060 and then added the cytostatic drug for an additional $24 \mathrm{~h}$ (Fig 4). Interestingly, we observed a reduced inhibition after this pre-treatment with NK1R antagonist resulting in less cytotoxicity from cisplatin and ifosfamide. Cisplatin alone induced $94.6 \pm 0.9 \%$ cell inhibition but only $83.1 \pm 1.9 \%$ cell inhibition, if cells were pre-incubated with L-733,060 at $\mathrm{IC}_{50}$ dose for $24 \mathrm{~h}$. The reduced inhibition achieved by the NK1R antagonist for ifosfamide application was even stronger, showing total cell death (100\%) for the cytostatic alone but only $85.9 \pm 2.0 \%$ when combined with L-733,060. Of note, this 
A

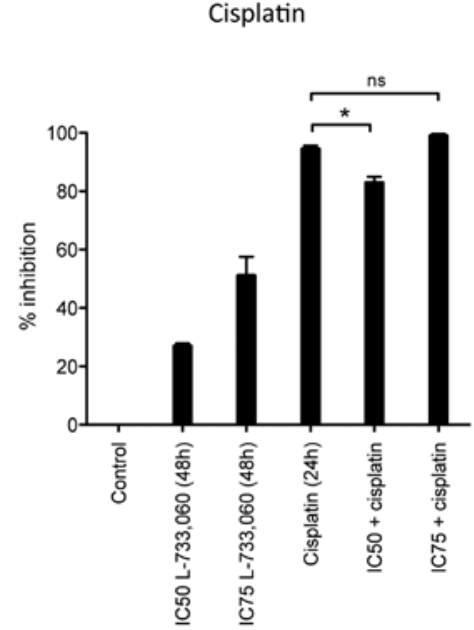

B

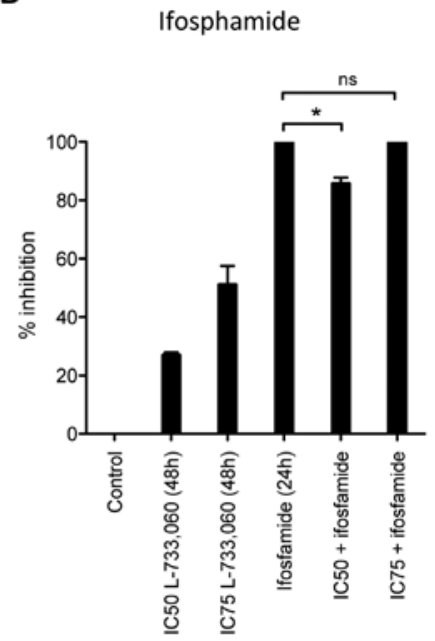

Figure 4. Pretreatment of non-malignant HEK293 cells with NK-1 receptor antagonists. In this experiment, cell inhibition is shown in percentage for HEK293 for different stimulation conditions. HEK293 cells were pre-incubated for $24 \mathrm{~h}$ with NK1R antagonist L-733,060. After this, (A) cisplatin and (B) ifosfamide were added for an additional $24 \mathrm{~h}$ and cytotoxicity assay was conducted as described. Error bars represent the SEM. ${ }^{*}<0.05 ;{ }^{* *} \mathrm{p}<0.01$ indicate statistical significance.

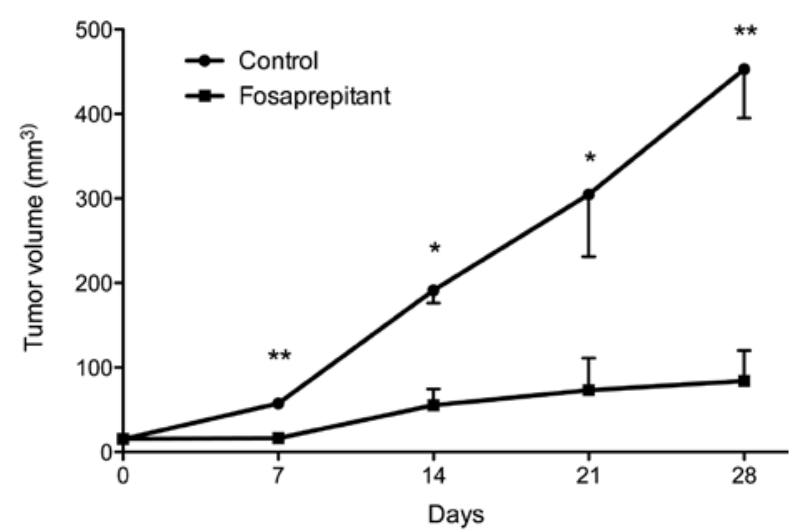

Figure 5. In vivo treatment of the human MG-63 xenografted mice with fosaprepitant. Nude mice were xenografted subcutaneously with human MG-63 cell line as described and randomly separated into two groups (control group, $\mathrm{n}=4$; treatment group, $\mathrm{n}=5$ ). Mice were checked daily and at the first sign of tumor growth, treatment was initiated with peritumoral s.c. injections of fosaprepitant (day 0 ) and weekly from thereon until day 21 . The tumor volume was measured and depicted as $\mathrm{mm}^{3}$ at the indicated time points. The error bars represent the SEM. ${ }^{*} \mathrm{p}<0.05 ;{ }^{* * *} \mathrm{p}<0.01$ indicate statistical significance.

effect was lost if higher concentrations $\left(\mathrm{IC}_{75}\right)$ of the $\mathrm{NK} 1 \mathrm{R}$ antagonist were used for pre-treatment.

Treatment of the human MG-63 mouse xenograft with fosaprepitant. Having observed a robust antitumor effect in vitro, we then studied the effect of NK1R antagonist fosaprepitant in vivo. Nude mice were xenografted with human MG-63 osteosarcoma cell line as described in Materials and methods and randomly separated into control and treatment groups once tumors became visible. Subsequently, treatments with peritumoral s.c. injections of fosaprepitant were started and were repeated weekly from thereon (days 0, 7, 14,21 and 28, see Fig. 5). The control group was injected with phosphate-buffered saline (PBS) at the exact same time points as the fosaprepitant injections. At each treatment point, measurement of tumor size
Table I. Histological analysis of in vivo treated osteosarcomas of human MG-63 xenografted mice.

\begin{tabular}{lccc}
\hline & Control & Treatment & P-value \\
\hline Necrosis & $18.75 \pm 10.3$ & $28.0 \pm 9.1$ & 0.1952 \\
Apoptosis & $7.5 \pm 3.5$ & $10.0 \pm 5.0$ & 0.4186 \\
Mitosis & $13.25 \pm 1.5$ & $14.4 \pm 3.3$ & 0.5415 \\
Nuclear grade & $2.25 \pm 0.5$ & $3.0 \pm 0.0$ & 0.6845 \\
Viability & $62.5 \pm 20.6$ & $45.0 \pm 19.2$ & 0.2253 \\
\hline
\end{tabular}

After termination of the in vivo experiments, mice were sacrificed and tumors were analyzed by a pathologist regarding the percentage of necrosis, apoptosis, mitosis, viability as well as nuclear grade. For each feature, $p$-value was calculated between the control and the treatment group.

was obtained as well as one week after the last injection (on day 28). The control group consisted of 4 mice, the treatment group consisted of 5 mice. At 7 days, tumor volume in the control group was $57.6 \pm 4.9$ and $16.4 \pm 4.3 \mathrm{~mm}^{3}$ in the treatment group $(\mathrm{p}<0.01)$. After 14 days, tumors in the control group were $191.4 \pm 15.2$ and $55.4 \pm 19.2 \mathrm{~mm}^{3}$ in the treatment group $(\mathrm{p}<0.05)$. At 21 and 28 days, the tumor volume was 304.7 \pm 53.6 and $452.9 \pm 57.7 \mathrm{~mm}^{3}$, respectively, in the control group and $73.2 \pm 38.2$ and $84.2 \pm 35.8 \mathrm{~mm}^{3}$, respectively, in the treatment group ( $\mathrm{p}<0.05$ at day 21 and $\mathrm{p}<0.01$ at day 28 ). Taken together, we saw a strong, statistically highly significant therapeutic effect in mice xenografted with the osteosarcoma cell line MG-63 after treatment with NK1R antagonist fosaprepitant.

Histology of treated mice. After completion of the in vivo experiments, the mice were sacrificed and their histologic features were analyzed regarding necrosis, apoptosis, mitosis, nuclear grade and viability (Table I). In the control group an average necrosis of $18.75 \pm 10.3 \%$ was found, compared to 
$28.0 \pm 9.1$ in the treatment group. Even though this marks a clear difference, no statistical significance was found $(\mathrm{p}=0.1952)$. The same is true for the rate of apoptosis, mitosis and nuclear grade (Table I). Viability in the control group was higher $(62.5 \pm 20.6 \%)$ compared to the treatment group $(45.0 \pm 19.2 \%)$, but due to the small sample size, differences were not statistically significant $(\mathrm{p}=0.2253)$. Histologic analysis of all tumor specimens clearly showed features of human osteosarcoma.

\section{Discussion}

Osteosarcoma, especially if metastases are present, is traditionally known to be a tumor with only modest long-term survival. Recent therapeutic changes and a multidisciplinary approach have increased the prognosis and close to two-thirds of patients can now be cured. Adjuvant chemotherapy has an established role in osteosarcoma in the control of subclinical metastatic disease. The most effective chemotherapeutic agents currently in use include high-dose methotrexate, doxorubicin, cisplatin and ifosfamide/etoposide (29). However, if metastatic spread has occurred, especially to other parts of the skeletal system, the prognosis is poor and survival is less than $20 \%$ (30). Therefore, additional therapeutic strategies are desperately required as part of future, multidisciplinary treatment approaches against osteosarcoma.

The SP/NK-1 receptor system has recently been identified as a potential antitumor target $(3,4)$. Blocking this receptor system with NK1R antagonists is currently under intense investigation for future anticancer strategies. To the best of our knowledge, until now nothing has been described regarding the expression of this receptor system in human osteosarcoma cell lines. Therefore, with the data presented here, for the first time we describe that NK1R is expressed in human osteosarcoma cell lines and that blocking of this receptor with non-peptide derived NK1R antagonists such as the clinical drug aprepitant and other NK1R antagonists results in robust tumor inhibition both in vitro and in vivo.

The NK1R is a tachykinin receptor with high affinity for the short neuropeptide SP, which is considered its natural ligand (31). Interestingly, it has been observed that presence of NK1R expression was related to the tumor cell subtype, with higher expression in the luminal human breast cancer cell lines expressing Her2 (SKBR3, BT474 and MDA-MD-453) than in the basal-like breast cancer cell lines (MDA-MB-468, MDA-MB-231, MCF10A and MCF12A). Moreover, the pro-apoptotic effect of NK1R inhibition was greater in cells with higher levels of NK1R expression, suggesting 'oncogenic addiction' of these cells to NK1R signaling (32).

Substantial efforts have been made to develop therapeutic inhibitors against NK1R in the last decade. Small molecules such as antagonists of NK1R represent an important opportunity to further exploit compounds that are active against this receptor as novel therapeutic agents (3). One promising compound is aprepitant (Emend), currently approved by the Food and Drug Administration (FDA) for the treatment of chemotherapyinduced nausea and vomiting and is available for oral use (33). It has also been successfully used as an off-label drug in the treatment of pain, migraine, emesis and certain psychiatric disorders. Fosaprepitant (Ivemend) is a water-soluble phosphoryl prodrug of aprepitant and is available for intravenous use $(19,22)$. In the present study, we demonstrated that treatment of the human osteosarcoma cell line with NK1R antagonists L-733,060, L-732,138 and aprepitant produced robust growth inhibition and cell death by apoptosis in vitro. On the other hand, exogenous SP induced cellular proliferation. When combining the two, we were able to show that cell proliferation induced by exogenous SP was partially reverted by administration of NK1R antagonists, suggesting a specific effect through the NK1R.

Our findings are in accordance with the recent understanding that NK1R antagonists have great antitumor potential against a large variety of human cancer cell lines such as human neuroblastoma, glioma, melanoma, retinoblastoma, pancreatic adenocarcinoma and many others (3). This observation suggests the possibility of a common mechanism for cancer cell proliferation mediated by SP and NK1R. If this was truly the case, targeting the NK1R with competing antagonists could potentially inhibit a large number of tumor cell types and tumors and could function as broad-spectrum antineoplastic drugs (3). While these observations are encouraging, it must be pointed out that at this early stage of investigation, little is known about possible tumor-escape mechanisms regarding the SP/NK-1 receptor system. Since this is the case with essentially all chemotherapeutic agents in a large variety of tumor types, under favorable circumstances, a single-target approach will most likely result in tumor-escape. Whether this will be the case with targeting the SP/NK-1 receptor system is impossible to predict at the moment, and further investigation needs to take into account this possibility when developing NK-1 receptor targeting anticancer drugs.

One of the most interesting findings among our results is our analysis of NK1R in HEK293 cells and its subsequent response to NK1R ligands. HEK 293 is a cell line derived from embryonic kidney tissue. This cell line is considered immortal, but benign. In our experiments, despite robust expression of the NK1R, cell inhibition with NK1R antagonists was significantly smaller compared to the MG-63 osteosarcoma cell line. This finding by itself endorses that the observed effects in vitro of NK1R antagonists are not general toxic effects. This becomes even clearer when interpreting our findings regarding the simultaneous treatment of cell lines in vitro with NK1R ligands and common cytostatic drugs. While there was a clear synergism between cytostatic drugs and NK1R antagonists in the case of a malignant human osteosarcoma cell line, this effect was not seen in the benign cell line HEK293. When the NK1R antagonist was given together with the cytostatic, the sole presence of the NK1R antagonists seemed to have a protective effect resulting in less cell inhibition compared to the cytostatic alone at least for some cytostatic drugs. Indeed and of utmost importance, compared to cytotoxicity in malignant cells, growth inhibition of the benign cell line HEK293 was only minimal. Whether this selectivity is exclusively due to the fact that one cell is malignant and the other benign, is impossible to determine from our results. Such a specific selectivity between malignant and benign cells has been previously hypothesized by others and was explained by overexpression of the NK1R in several malignant cells compared to benign cells (20). In our study, we clearly see a correlation between the relative gene expression and the response rate of tumor growth to NK1R antagonists. Therefore, our data provided here support this concept. It will be interesting and necessary to investigate in closer detail whether 
targeting the NK1R truly results in selectivity for malignant tissue, and what additional mechanisms are responsible.

An in vivo anticancer effect for NK1R antagonist has previously been reported by Palma et al (26). In their study, nude mice xenografted subcutaneously with malignant glioma cell line U373 MG showed significant reduction of tumor size after treatment with NK1R antagonists. This therapeutic effect could be achieved both with intravenous and s.c. treatment and was partially reversible with administration of SP. In another study by Bigioni et al a similar in vivo effect was observed for treatment with NK1R antagonists in the human breast carcinoma cell line MDA-MB-231 xenografted mice. Interesting in their study was the observation that in vivo targeting of both NK1R and NK-2 resulted in a therapeutic effect (27). In accordance with these findings, in our study, we observed a stunning therapeutic effect for osteosarcoma xenografted nude mice. However, we have currently no understanding on which method of application is ideal for treatment of xenografted mice, and whether conclusions of such experiments in animal models can be drawn regarding possible treatment approaches in humans. We used peritumoral s.c. injections as the mode of application, which was therefore different from intravenous injection used by Palma et al (26) and Bigioni et al (27). Nonetheless, all methods of application resulted in a therapeutic effect.

It is known that those osteosarcomas which show relatively low degrees of necrosis after administration of chemotherapy have poorer survival than patients with more chemotherapy-responsive tumors $(29,30)$. In our study, we observed histologic changes similar to those found after chemotherapy in osteosarcoma treatment. Even though no statistical differences could be calculated between the two groups, our findings clearly show a higher rate of necrosis and apoptosis paired with a decreased rate of viable cells in the histology of the treatment group compared to the control group (Table I). Whether NK1R can have a role as anticancer agent in those osteosarcomas that show low response rates to chemotherapy is impossible to say from our data and requires further, intense investigation.

In summary, we have demonstrated for the first time that human osteosarcoma cell line MG-63 expresses the NK1R and that the clinical drug aprepitant and other NK1R antagonists can inhibit cell growth and induce apoptosis both in vitro and in vivo in this tumor. This effect was specifically mediated through the NK1R, and selectivity was observed for malignant cells compared to benign cells. Also, NK1R antagonist showed a synergistic effect on osteosarcoma cells when combined with common cytostatics but not on benign HEK 293 cells. Despite these encouraging results presented here, many questions remain unanswered. This includes the important question on whether the SP/NK-1 receptor system is ubiquitously expressed in tumor cells and represents a universal survival pathway in cancer cells, whether targeting of NK1R leads to tumor escape mechanisms by the tumor cell and whether overexpression of NK1R is entirely responsible for the observed selectivity between malignant and benign cells, thereby, giving hints on what type of tumors might be treated with these antagonists. Nevertheless, our result clearly identify the NK1R as a promising therapeutic target in osteosarcoma and NK1R antagonists should be further explored for the use in future anticancer strategies in patients that suffer from this cancer.

\section{Acknowledgements}

We thank Raquel Gómez from the Instituto de Biomedicina de Sevilla (IBIS) for technical assistance when performing and analyzing western blot experiments. Michael Berger was supported by the German Academic Exchange Program (DAAD).

\section{References}

1. Wittig JC, Bickels J, Priebat D, et al: Osteosarcoma: a multidisciplinary approach to diagnosis and treatment. Am Fam Physician 65: 1123-1132, 2002.

2. Ritter J and Bielack SS: Osteosarcoma. Ann Oncol 21 (Suppl 7): vii320-vii325, 2010.

3. Muñoz M, Rosso $M$ and Coveñas R: A new frontier in the treatment of cancer: NK-1 receptor antagonists. Curr Med Chem 17: 504-516, 2010.

4. Muñoz M, Rosso M and Coveñas R: The NK-1 receptor: a new target in cancer therapy. Curr Drug Targets 12: 909-921, 2011.

5. Vanden Broeck J, Torfs H, Poels J, et al: Tachykinin-like peptides and their receptors. A review. Ann NY Acad Sci 897: 374-387, 1999.

6. Patacchini R and Maggi CA: Peripheral tachykinin receptors as targets for new drugs. Eur J Pharmacol 429: 13-21, 2001.

7. Rollandy I, Dreux C, Imhoff V and Rossignol B: Importance of the presence of the N-terminal tripeptide of substance $\mathrm{P}$ for the stimulation of phosphatidylinositol metabolism in rat parotid gland: a possible activation of phospholipases C and D. Neuropeptides 13: 175-185, 1989.

8. Pradier L, Heuillet E, Hubert JP, et al: Substance P-evoked calcium mobilization and ionic current activation in the human astrocytoma cell line U 373 MG: pharmacological characterization. J Neurochem 61: 1850-1858, 1993.

9. Luo W, Sharif TR and Sharif M: Substance P-induced mitogenesis in human astrocytoma cells correlates with activation of the mitogen-activated protein kinase signaling pathway. Cancer Res 56: 4983-4991, 1996.

10. DeFea KA, Zalevsky J, Thoma MS, et al: Beta-arrestindependent endocytosis of proteinase-activated receptor 2 is required for intracellular targeting of activated ERK1/2. J Cell Biol 148: 1267-1281, 2000.

11. Palma C: Tachykinins and their receptors in human malignancies. Curr Drug Targets 7: 1043-1052, 2006.

12. Muñoz M, González-Ortega A and Coveñas R: The NK-1 receptor is expressed in human leukemia and is involved in the antitumor action of aprepitant and other NK-1 receptor antagonists on acute lymphoblastic leukemia cell lines. Invest New Drugs 30: 529-540, 2012.

13. Muñoz $M$, Rosso $M$, Pérez $A$, et al: The NK1 receptor is involved in the antitumoural action of L-733,060 and in the mitogenic action of substance $\mathrm{P}$ on neuroblastoma and glioma cell lines. Neuropeptides 39: 427-432, 2005.

14. Muñoz M, Rosso M, Pérez A, et al: Antitumoral action of the neurokinin-1-receptor antagonist L-733,060 and mitogenic action of substance $\mathrm{P}$ on human retinoblastoma cell lines. Invest Ophthalmol Vis Sci 46: 2567-2570, 2005

15. Muñoz M, Rosso $M$ and Coveñas $R$ : The NK-1 receptor is involved in the antitumoural action of L-733,060 and in the mitogenic action of substance $\mathrm{P}$ on human pancreatic cancer cell lines. Lett Drug Des Discov 3: 323-329, 2006.

16. Muñoz M, Rosso M, Aguilar F, et al: NK-1 receptor antagonists induce apoptosis and counteract substance P-related mitogenesis in human laryngeal cancer cell line HEp-2. Invest New Drugs 26: 111-118, 2008.

17. Rosso M, Robles-Frias MJ, Coveñas R, Salinas-Martín MV and Muñoz M: The NK-1 receptor is expressed in human primary gastric and colon adenocarcinomas and is involved in the antitumor action of L-733,060 and the mitogenic action of substance $\mathrm{P}$ on human gastrointestinal cancer cell lines. Tumour Biol 29: 245-254, 2008.

18. Muñoz M, Pérez A, Rosso M, et al: The NK-1 receptor is expressed in human melanoma and is involved in the antitumor action of the NK-1 receptor antagonist aprepitant on melanoma cell lines. Lab Invest 90: 1259-1269, 2010. 
19. Navari RM: Fosaprepitant (MK-0517): a neurokinin-1 receptor antagonist for the prevention of chemotherapy-induced nausea and vomiting. Expert Opin Investig Drugs 16: 19771985, 2007.

20. Muñoz M, Rosso M: The NK-1 receptor antagonist aprepitant as a broad spectrum antitumor drug. Invest New Drugs 28 : 187-193, 2010.

21. Quartara L, Altamura M, Evangelista S and Maggi CA: Tachykinin receptor antagonists in clinical trials. Expert Opin Investig Drugs 18: 1843-1864, 2009.

22. Quartara L and Altamura M: Tachykinin receptors antagonists: from research to clinic. Curr Drug Targets 7: 975-992, 2006.

23. Muñoz M, Pérez A, Rosso M, Zamarriego C and Rosso R: Antitumoral action of the neurokinin-1 receptor antagonist L-733 060 on human melanoma cell lines. Melanoma Res 14 183-188, 2004.

24. Muñoz M, Rosso M, Casinello F and Coveñas R: Paravertebral anesthesia: how substance P and the NK-1 receptor could be involved in regional block and breast cancer recurrence. Breast Cancer Res Treat 122: 601-603, 2010.

25. Muñoz $M$ and Coveñas R: Neurokinin-1 receptor: a new promising target in the treatment of cancer. Discov Med 10: 305-313, 2010

26. Palma C, Bigioni M, Irrissuto C, et al: Anti-tumour activity of tachykinin NK1 receptor antagonists on human glioma U373 MG xenograft. Br J Cancer 82: 480-487, 2000.
27. Bigioni M, Benzo A, Irrissuto C, Maggi CA and Goso C: Role of NK-1 and NK-2 tachykinin receptor antagonism on the growth of human breast carcinoma cell line MDA-MB-231. Anticancer Drugs 16: 1083-1089, 2005.

28. Muñoz M, Rosso M, Pérez A, et al: Neurokinin-1 receptors located in human retinoblastoma cell lines: antitumor action of its antagonist, L-732,138. Invest Ophthalmol Vis Sci 48: 2775-2781, 2007.

29. Ayerza MA, Farfalli GL, Aponte-Tinao L and Muscolo DL: Does increased rate of limb-sparing surgery affect survival in osteosarcoma? Clin Orthop Relat Res 468: 2854-2859, 2010.

30. Weber K, Damron TA, Frassica FJ and Sim FH: Malignant bone tumors. Instr Course Lect 57: 673-688, 2008.

31. Hökfelt T, Pernow B and Wahren J: Substance P: a pioneer amongst neuropeptides. J Intern Med 249: 27-40, 2001.

32. Almendro V, García-Recio S and Gascón P: Tyrosine kinase receptor transactivation associated to $G$ protein-coupled receptors. Curr Drug Targets 11: 1169-1180, 2010.

33. Tattersall FD, Rycroft W, Cumberbatch M, et al: The novel NK1 receptor antagonist MK-0869 (L-754,030) and its water soluble phosphoryl prodrug, L-758,298, inhibit acute and delayed cisplatin-induced emesis in ferrets. Neuropharmacology 39: 652-663, 2000 